\title{
Caracterização física, físico-química e centesimal do fruto de jatobá-do-cerrado (Hymenaea stigonocarpa Mart.)
}

\section{Physical, physicochemical and centesimal characterization of jatobá-do-cerrado (Hymenaea stigonocarpa Mart.)}

\author{
Edilene Ferreira da Silva ${ }^{(\mathbb{D}}{ }^{1}$, Elisabeth Mariano Batista ${ }^{\left(D^{2}\right.}{ }^{2}$, Érica Jamilly do Nascimento Almeida (D) $^{3}$, Lúcia $^{2}$ \\ Mara dos Reis Lemos ${ }^{4}{ }^{4}$, Samuel Carneiro de Barcelos ${ }^{5}{ }^{5}$, Pahlevi Augusto de Souza ${ }^{6}$
}

\begin{abstract}
${ }^{1}$ Doutoranda em Ciência dos Alimentos, Universidade Federal de Santa Catarina, Florianópolis. +558699962-9303. E-mail: silvaedilene16@hotmail.com. ${ }^{2}$ Doutoranda em Ciência e Tecnologia de Alimentos, Universidade Federal do Ceará, Fortaleza. E-mail: elisabethmariano@ hotmail.com. ${ }^{3}$ Doutoranda em Ciência dos Alimentos, Universidade Federal de Santa Catarina, Florianópolis. E-mail: lucia_mara15@hotmail.com. ${ }^{4}$ Mestre em Tecnologia em Alimentos, Instituto Federal de Ciência e Tecnologia do Ceará-Campus Limoeiro do Norte. E-mail: ericaalmeida.nutri@gmail.com. ${ }^{5}$ Doutorando em Biotecnologia, Rede Nordeste de Biotecnologia, Universidade Estadual do Ceará, Сampus Itaperi, Fortaleza. E-mail: samuelbarcelos05@gmail.com. ${ }^{6}$ Professor Doutor em Fitotecnia, Instituto Federal de Educação, Ciência e Tecnologia do Rio Grande do Norte, Currais Novos. E-mail: pahlevi10@ hotmail.com.
\end{abstract}

\section{A R T I G O}

Recebido: 10/09/2019

Aprovado: $13 / 02 / 2020$

\section{Palavras-chave:}

Casca

Composição-química

Farinha

Semente

Key words:

Bark

Chemical composition

Flour

Seed

\section{R E S U M O}

Dentre as espécies nativas do Cerrado está o jatobá, uma leguminosa arbórea que possui um fruto, em que sua polpa farinácea apresenta forte potencial para o enriquecimento de preparações. Objetivou-se avaliar as características físicas do fruto de jatobá-do-cerrado (Hymenaea stigonocarpa Mart.), e as características físico-químicas e composição centesimal na polpa, semente e casca do fruto. A polpa do jatobá foi homogeneizada e denominada de Farinha da Polpa (FP). As sementes e as cascas do jatobá foram maceradas e reduzidas e, em seguida, foram trituradas em liquidificador industrial, obtendo-se as farinhas denominadas de Farinha da Semente (FS) e Farinha da Casca (FC). Foi avaliado as variáveis biométricas das sementes e dos frutos do jatobá. Os resultados obtidos validam a possibilidade da FP e FS serem utilizadas como ingrediente no enriquecimento proteico de alimentos, por apresentar quantidade expressiva desse componente. Além disso, a FP e FC apresentaram potencial de aplicação em alimentos que contenham baixo teor de fibras, pois exibiram resultados expressivos para fibra bruta (17,82 e $38,55 \%$, respectivamente). Todas as partes analisadas do fruto obteve fontes consideráveis de nutrientes, possibilitando a sua utilização na elaboração de novos produtos alimentícios, pois apresentaram elevados teores de proteínas, carboidratos e fibras.

\section{A B S T R A C T}

Among the native species of the Cerrado is the jatobá, a tree legume that has a fruit, in which its flour pulp has strong potential for enriching preparations. The objective was to evaluate the physical characteristics of the fruit of jatobá-do-cerrado (Hymenaea stigonocarpa Mart.), And the physical-chemical characteristics and centesimal composition in the pulp, seed and skin of the fruit. The jatobá pulp was homogenized and called Pulp Flour (FP). The seeds and husks of the jatoba were macerated and reduced and then crushed in an industrial blender, obtaining the flours called Seed Flour (FS) and Shell Flour (FC). The biometric variables of the seeds and fruits of the jatoba were evaluated. The results obtained validate the possibility of FP and FS being used as an ingredient in the protein enrichment of foods, due to the significant amount of this component. In addition, FP and FC showed potential for application in foods that contain low fiber content, as they showed expressive results for crude fiber (17,82 and 38,55\%, respectively). All the analyzed parts of the fruit obtained considerable sources of nutrients, enabling their use in the preparation of new food products, as they presented high levels of proteins, carbohydrates and fibers.

\author{
Revista Verde \\ ISSN 1981-8203 \\ Pombal, Paraíba, Brasil
}

v. 15, n.2, abr.-jun., p.139-145, 2020

doi: $10.18378 /$ rvads.v15i2.6992 


\section{INTRODUÇÃO}

O Cerrado é o segundo maior bioma do Brasil e da América do Sul, perdendo em extensão somente para a Floresta Amazônica. O uso da biodiversidade nativa do cerrado tem grande importância para populações rurais, e seu manejo sustentável possui grande potencial para se tornar elemento estratégico à conservação dos ecossistemas (CARDOSO, 2011). Diversas árvores nativas do Cerrado produzem frutos cujas características sensoriais são altamente apreciadas pela população. Esses frutos exercem importante papel econômico, visto que contribuem, através da produção de subprodutos como farinhas e biscoitos por exemplo, para a geração de renda (REIS, SCHMIELE, 2019; COHEN, 2010).

Graças a sua localização geográfica e extensão, o Cerrado conta com uma ampla diversidade de vegetais que possuem potencial de utilização para diversos fins, inclusive para uso na dieta humana. As fruteiras nativas ocupam lugar importante no ecossistema do Cerrado e seus frutos, que são comercializados em feiras na região, apresentam boa aceitação popular (ARRUDA et al., 2015; MACIEL et al., 2016). Os mesmos oferecem sabores únicos e elevados teores de açúcares, vitaminas, proteínas e sais minerais, podendo ser consumidos in natura ou na forma de sucos, licores, sorvetes e geleias (SOUSA et al., 2012).

Dentre as espécies nativas do Cerrado está o jatobá, uma leguminosa arbórea que possui um fruto com comprimento entre 6 e $18 \mathrm{~cm}$ e diâmetro de 3 a $6 \mathrm{~cm}$; sua polpa farinácea, de cor creme, envolve de 3 a 6 sementes; sua árvore é uma espécie tropical que mede de 4 a 6 metros de altura, sendo mais encontrada em terreno seco, muitas vezes, de pouca fertilidade. A polpa farinácea do jatobá apresenta forte potencial para o enriquecimento de preparações que utilizam farinhas, tais como bolos, pães e biscoitos (SILVA, 2013).

Pesquisas demonstram o potencial nutricional da farinha do jatobá na utilização do desenvolvimento de formulações, para substituir a farinha de trigo, visando incluir na dieta de indivíduos saudáveis e pessoas com doenças crônicas, como diabéticos (MACIEL et al., 2016; RAMOS et al., 2018; SILVA et al., 2019). Além disso, sua composição nutricional tem despertado interesse por apresentarem excelentes propriedades que as tornam importantes no desenvolvimento de novos produtos alimentícios e farmacêuticos (MENEZES FILHO et al., 2019).

Diante disto, objetivou-se avaliar as características físicas do fruto de jatobá-do-cerrado (Hymenaea stigonocarpa Mart.), as características físico-químicas e composição centesimal da polpa, semente e casca do fruto.

\section{MATERIAL E MÉTODOS}

\section{Obtenção dos frutos}

Os jatobás-do-cerrado (Hymenaea stigonocarpa Mart.) foram colhidos em fase de maturidade fisiológica com coloração da casca uniforme, marrom escuro, sem fissuras, íntegros, no município de União, Piauí, Brasil (região Nordeste), que também faz parte do cerrado, latitude $04^{\circ} 35^{\prime}$ 09 " S, longitude $42^{\circ} 15$ ' 51 " W, altitude $52 \mathrm{~m}$, área 1182,2 $\mathrm{Km}^{2}$ e transportados em transporte próprio dos autores, em caixas térmicas, até a cidade de Limoeiro do Norte, Ceará, Brasil. Em seguida, foram encaminhados ao Laboratório de Processamento de Frutas e Hortaliças do Instituto Federal de Educação, Ciência e Tecnologia do Ceará (IFCE), campus Limoeiro do Norte, onde foram lavados em água corrente e em seguida sanitizados com solução clorada na concentração de 50 ppm por 15 minutos.

\section{Obtenção das farinhas}

Foram processados ao todo 20 frutos de jatobás-docerrado, retirando as cascas com martelo de aço inox, para remover a polpa e semente, sendo que cada parte do fruto foi reservada em béquer de plástico com capacidade de $1 \mathrm{~L}$. As polpas foram separadas das sementes, com auxílio de uma faca, e homogeneizada em liquidificador doméstico (Mallory, Tornado-B91201262), por aproximadamente 15 minutos, obtendo assim a Farinha da Polpa, denominada de FP. As sementes e as cascas foram maceradas com auxílio de um martelo e posteriormente trituradas em liquidificador industrial (Metalúrgica VISA LTDA-LQ25) por aproximadamente 15 minutos e peneiradas em peneira $\mathrm{n}^{\circ} 60$, com $250 \mu \mathrm{m}$ de abertura, obtendo-se as farinhas denominadas de Farinha da Semente $=$ FS e Farinha da Casca $=$ FC.

\section{Caracterização física do fruto}

$\mathrm{Na}$ Biometria, mediu-se o comprimento, largura e espessura do fruto inteiro e de sua semente, utilizando um paquímetro (Stehmeyer-FMS), sendo os resultados expressos em mm para cada parâmetro biométrico. As medidas foram realizadas em sextuplicata.

Para determinar o rendimento, pesou-se o fruto inteiro, a casca, a polpa e a semente, em balança analítica (BEL Engineering-M214A). O rendimento foi realizado em sextuplicata.

\section{Caracterização físico-química das farinhas}

Foram realizadas análises físico-químicas das farinhas da casca, polpa e semente do jatobá-do-cerrado, onde foram determinados: $\mathrm{pH}$ em potenciômetro de bancada (Lucadema, Luca-210) previamente calibrado com soluções tampão de $\mathrm{pH}$ 4,0 e 7,0, com $10 \mathrm{~g}$ da amostra diluída em $100 \mathrm{~mL}$ de água destilada; sólidos solúveis, com 10 g da amostra diluída em 100 $\mathrm{mL}$ de água destilada e, posteriormente o diluído filtrado em algodão e realizado a leitura em refratômetro digital de bancada (Abbe Refratometer, Optronics-WYA-1S), os resultados foram expressos em ${ }^{\circ}$ Brix; acidez titulável por titulação (utilizando 10 g de amostra) com solução de $\mathrm{NaOH}(0,1 \mathrm{M})$ e fenolftaleína (1\%) como indicador e os resultados expressos em \% de ácido cítrico. Estas análises foram realizadas em triplicata, de acordo com os métodos descrito pelo Instituto Adolfo Lutz (2008).

A determinação de vitamina $\mathrm{C}$ foi realizada em triplicata, por método titulométrico, de acordo com Strohecker e Henning (1967), utilizando $5 \mathrm{~g}$ da amostra e os resultados expressos em mg.100 g-1 de ácido ascórbico.

\section{Caracterização centesimal das farinhas}

As análises de composição centesimal (realizadas em base seca) foram: Matéria seca $\left(\mathrm{g} .100 \mathrm{~g}^{-1}\right)$ por meio da secagem em estufa com circulação de ar à temperatura de $105{ }^{\circ} \mathrm{C}$ até peso 
constante; Proteínas (g. $100 \mathrm{~g}^{-1}$ ) estimada com base no teor total de nitrogênio, determinado pelo método de Micro-Kjeldahl, usando fator de conversão de 6,25; Cinzas $\left(\mathrm{g} .100 \mathrm{~g}^{-1}\right)$ por incineração em mufla a $550{ }^{\circ} \mathrm{C}$; Lipídios $\left(\mathrm{g} .100 \mathrm{~g}^{-1}\right)$ em equipamento de refluxo (extrator tipo Soxhlet) com solvente hexano, durantes seis horas. As análises foram realizadas em triplicata, conforme metodologia descrita pelo Instituto Adolfo Lutz (2008).

Determinou-se o teor de fibra bruta em triplicata, através da digestão da amostra em solução de $\mathrm{H}_{2} \mathrm{SO}_{4}$ por 40 minutos, seguida de $\mathrm{NaOH}$ por mais 40 minutos, de acordo com a Association of Official Analytical Chemists (2000).

Carboidrato $\left(\mathrm{g} .100 \mathrm{~g}^{-1}\right)$ por diferença $=100-($ umidade + cinzas + proteínas + lipídeos) - fibras; Valor calórico total (VCT) em Kcal.100 $\mathrm{g}^{-1}$ foi calculado pela soma dos resultados das multiplicações dos valores encontrados de proteína, lipídios e carboidrato total (por diferença, incluindo as fibras) pelos seus respectivos fatores de conversão de Atwater (4, 9 e $4 \mathrm{kcal}$ ), de acordo com o seguinte cálculo: $\mathrm{VCT}^{(\mathrm{Kcal})}=($ Proteína $\mathrm{x} 4)+$ (Carboidratos $\mathrm{x} 4)+($ Lipídios $\mathrm{x}$ 9). As análises foram realizadas em triplicata, conforme metodologia descrita pelo Instituto Adolfo Lutz (2008).

\section{Análise estatística}

Foram verificados os pressupostos de normalidade (Shapiro-Wilk) e homogeneidade (Bartlett) das variáveis, todas as variáveis estudadas apresentaram distribuição normal e homogeneidade. Foi utilizado a Análise de Variância seguido de pós-teste pelo teste de Tukey, com a comparação entre os pares de médias $(\mathrm{p}<0,05)$. O software estatístico utilizado para as análises foi o programa estatístico R, versão 3.5.0 (R CORE TEAM, 2018). Quando aplicado, os dados das análises de caracterização biométrica e rendimento foram tratados como média e desvio padrão.

\section{RESULTADOS E DISCUSSÃO}

\section{Caracterização física do fruto}

Pode ser observado que o fruto do jatobá-do-cerrado apesentou um maior comprimento, largura e espessura, respectivamente (Figura 1). Com características alongadas, ápice arredondado ou levemente retuso, base arredondada e margem inteira ou levemente ondulada.

Figura 1. Fruto inteiro do jatobá-do-cerrado (Hymenaea stigonocarpa Mart.) proveniente do município de União, Piauí.

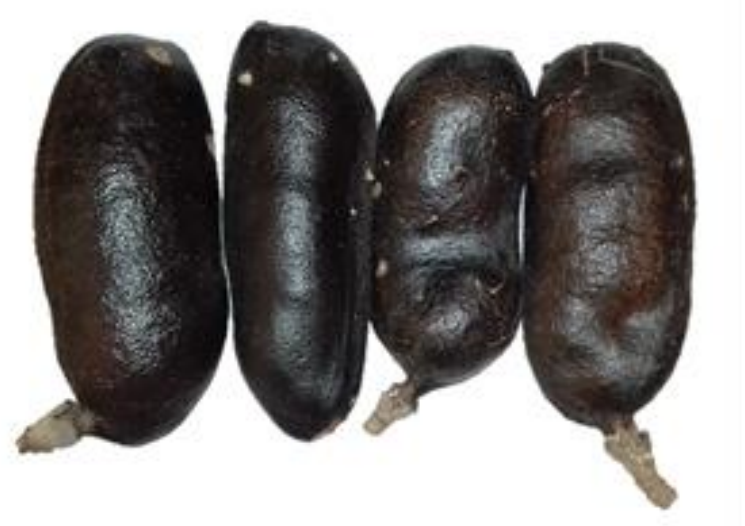

Fonte: Autores (2016).
As sementes (Figura 2) também apresentaram o mesmo comportamento do fruto do jatobá-do-cerrado, apresentando um maior comprimento, largura e espessura, respectivamente. Cunha-Silva et al. (2012) em sua pesquisa quanto à forma, as espécies estudadas apresentaram sementes com forma predominantemente achatadas, além de sementes elipsoides, oblongas e arredondadas, semelhantes aos dados encontrados no presente estudo, nas mesmas características.

Figura 2. Semento do fruto inteiro do jatobá-do-cerrado (Hymenaea stigonocarpa Mart.) proveniente do município de União, Piauí.

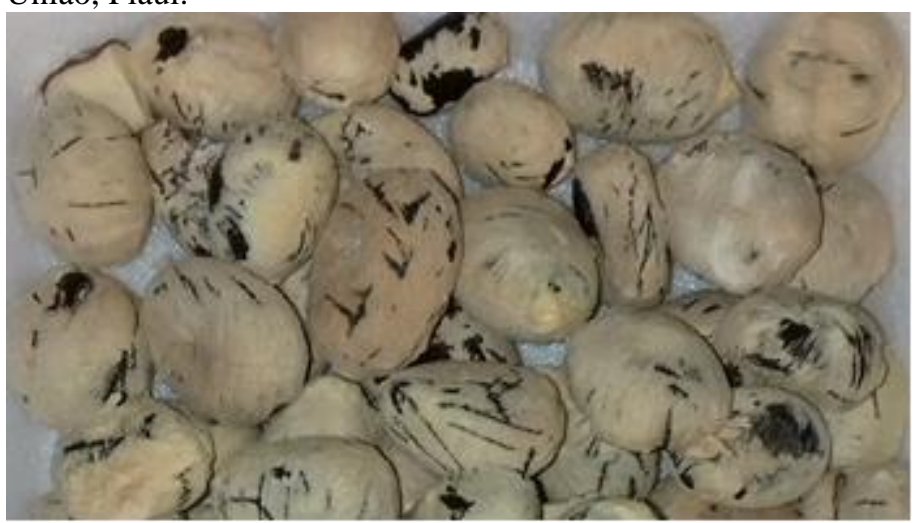

Fonte: Autores (2016).

A Figura 3 mostra a parte interna do fruto do Jatobá, sua polpa, apresentando fruto em formato de vagem, com casca amadeirada marrom escura e polpa de cor amarela creme. Dias et al. (2013) descreve que o sabor e aroma do fruto é adocicado, suas sementes são achatadas e de cor marrom escura, características físicas de acordo com o encontrado na caracterização do fruto do presente trabalho.

Figura 3. Fruto inteiro do jatobá-do-cerrado (Hymenaea stigonocarpa Mart.), apresentando sua polpa proveniente do município de União, Piauí.

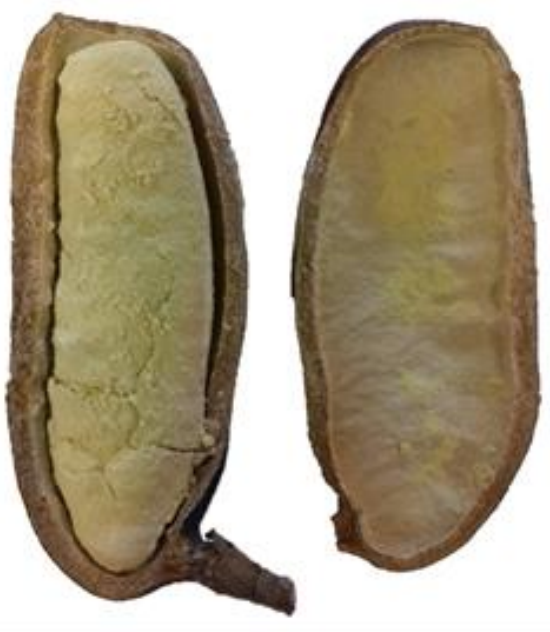

Fonte: Autores (2016).

Os valores biométricos encontrados no presente trabalho (Tabela 1) foram superiores aos avaliados por Dias et al. (2013), que obteve médias do fruto de $9,34 \mathrm{~cm}$ para o comprimento; $4,68 \mathrm{~cm}$ para a largura e $4,11 \mathrm{~cm}$ para a espessura. Os mesmos autores também analisaram a semente do fruto, e obtiveram 
valores de 2,32; 1,98 e $1,11 \mathrm{~cm}$ para comprimento, largura e espessura, respectivamente.

Tabela 1. Caracterização biométrica (média \pm DP) ${ }^{1}$ do fruto Jatobá-do-Cerrado (Hymenaea stigonocarpa Mart.).

\begin{tabular}{lccc}
\hline Amostras & Largura $(\mathrm{mm})$ & $\begin{array}{c}\text { Comprimento } \\
(\mathrm{mm})\end{array}$ & $\begin{array}{c}\text { Espessura } \\
(\mathrm{mm})\end{array}$ \\
\hline Fruto inteiro & $53,53 \pm 8,77$ & $131,97 \pm 10,47$ & $23,46 \pm 9,02$ \\
Semente & $19,99 \pm 1,44$ & $26,80 \pm 1,53$ & $14,22 \pm 1,49$ \\
\hline
\end{tabular}

$\left.{ }^{1}\right)$ Média de seis repetições \pm desvio padrão.

Cunha-Silva et al. (2012), analisaram as características físicas do jatobá, e encontraram média de $25,7 \mathrm{~mm}$ de comprimento; $18,7 \mathrm{~mm}$ de largura e 11,4 $\mathrm{mm}$ de espessura.

Os resultados do rendimento do processamento do jatobádo-cerrado (Tabela 2) revelaram que a casca é a principal responsável pelo peso do fruto, com valor médio de $72,36 \mathrm{~g}$, que representa $70,12 \%$ do fruto inteiro, enquanto a polpa é o constituinte com menor peso, com 11,69 g (11,32\%), e a semente com 19,14 g (18,54\%).

Tabela 2. Rendimento (média $\pm \mathrm{DP})^{1}$ da polpa, semente e casca do jatobá-do-cerrado (Hymenaea stigonocarpa Mart.).

\begin{tabular}{lcc}
\hline \multicolumn{1}{c}{ Amostras } & Peso $(\mathrm{g})^{1}$ & $\begin{array}{c}\text { Rendimento em } \\
\%^{2}\end{array}$ \\
\hline Fruto inteiro & $107,16 \pm 30,73$ & 100 \\
Polpa & $11,69 \pm 1,22$ & 70,12 \\
Semente & $19,14 \pm 5,32$ & 11,32 \\
Casca & $72,36 \pm 27,0$ & 18,54 \\
\hline
\end{tabular}

$\left.{ }^{1}\right)$ Média de seis repetições \pm desvio padrão; $\left({ }^{2}\right)$ Média de seis repetições.
Cardoso et al. (2013) analisaram as características físicas do fruto do jatobá e também observaram que a casca corresponde à maior parte do fruto, tendo encontrado, no entanto, valores menores para o rendimento que os obtidos pelo presente estudo para todos os constituintes do fruto, reportando valores de 49,7 g para o fruto inteiro, 8,4 g para a polpa, 13,9 g para a semente e $27,3 \mathrm{~g}$ para a casca. Cunha-Silva et al. (2012), encontraram para o fruto inteiro do jatobá um peso de $119,34 \mathrm{~g}$ e para as sementes de 5,71 g, também relatam que a análise comparativa entre as diferentes espécies do gênero Hymenaea mostrou que Hymenaea intermedia, encontrada na floresta Amazônica, possui frutos grandes que se destacaram em todos os parâmetros biométricos avaliados.

Dias et al. (2013) estudando os frutos (Hymenaea courbaril L.) provenientes dos estados de São Paulo e Minas Gerais, obtiveram valores médios de 63,86 g para o fruto inteiro e 3,74 g para a semente, dados esses discrepantes dos encontrados na presente pesquisa, revelando a importância da caracterização dos frutos em diferentes regiões.

Logo, podemos verificar que o rendimento da polpa é baixo. No entanto, as outras partes do fruto possuem potencial de exploração, como exemplo, a utilização da casca para extração de compostos naturais (PUGA, 2015).

\section{Caracterização físico-química das farinhas}

Os valores de $\mathrm{pH}$ das farinhas do presente trabalho variaram de 5,04 para a FC a 5,67 para a FS (Tabela 3). Os valores de $\mathrm{pH}$ das farinhas apresentaram diferença estatística ( $p$ $<0,05)$ entre si, a FC apresentou o menor valor de $\mathrm{pH}$.

Tabela 3. Análises físico-químicas (média $\pm \mathrm{DP})^{1}$ da farinha da polpa, semente e casca do jatobá-do-cerrado (Hymenaea stigonocarpa Mart.).

\begin{tabular}{ccccc}
\hline \multirow{2}{*}{ Farinha } & $\mathrm{pH}$ & Acidez & Parâmetros \\
\cline { 2 - 5 } & $5,41 \pm 0,02 \mathrm{~b}$ & $1,48 \pm 0,10 \mathrm{a}$ & Sólidos Solúveis $\left({ }^{\circ}\right.$ Brix $)$ & $\begin{array}{c}\text { Vitamina C } \\
\left(\mathrm{mg}^{10} 100 \mathrm{~g}^{-1}\right)\end{array}$ \\
\hline Polpa & $5,67 \pm 0,03 \mathrm{a}$ & $0,41 \pm 0,03 \mathrm{~b}$ & $52,4 \pm 0,91 \mathrm{a}$ & $17,77 \pm 1,92 \mathrm{a}$ \\
Semente & $5,04 \pm 0,01 \mathrm{c}$ & $0,49 \pm 0,04 \mathrm{~b}$ & $13,8 \pm 0,6 \mathrm{~b}$ & $12,77 \pm 0,96 \mathrm{~b}$ \\
Casca & $8,4 \pm 0,6 \mathrm{c}$ & $11,11 \pm 0,96 \mathrm{~b}$ \\
\hline
\end{tabular}

( $\left.{ }^{1}\right)$ Média de três repetições \pm desvio padrão. a, b, c, Letras minúsculas distintas na mesma coluna indicam diferença significativa (p < 0,05$)$ pelo teste de Tukey.

Os valores apresentados para esse parâmetro por todas as amostras analisadas estiveram acima do valor de $\mathrm{pH} 4,00$ considerado eficaz contra a proliferação da grande maioria dos microrganismos patogênicos, uma vez que alimentos de baixa acidez (pH > 4,50) são os mais sujeitos a multiplicação microbiana, tanto de espécies patogênicas quanto de espécies deteriorantes, de acordo com Fiorda e Siqueira (2009). Sendo assim, todos os constituintes do jatobá estão sujeitos ao desenvolvimento desses microrganismos, necessitando de processamento e armazenamento adequados, quanto as condições higiênico-sanitárias.

Pereira et al. (2016) elaboraram uma farinha da polpa do jatobá para a utilização em substituição parcial a farinha de trigo, no desenvolvimento de biscoitos do tipo amanteigados e encontraram um valor de $\mathrm{pH}$ de 5,92, próximo ao resultado obtido na presente pesquisa.

$\mathrm{O}$ teor de acidez das farinhas da semente e casca foram inferiores estatisticamente $(p<0,05)$ ao encontrado na polpa, que pode ser explicado devido a presença dos constituintes, que variam de acordo os fatores endofoclimaticos e estádio de maturação dos frutos, conforme explicado por Sousa et al. (2012).

A respeito dos sólidos solúveis, houve diferença estatística entre o teor presente nas FP, FS e FC, sendo que a FP se destacou pelo maior valor, com 52,4 ${ }^{\circ}$ Brix, característica esperada, pois segundo Cohen (2010), o Jatobá apresenta-se como uma das espécies frutíferas do Cerrado com mais alto teor de açúcares (COHEN, 2010).

Os resultados obtidos para vitamina $\mathrm{C}$ (ácido ascórbico em $\left.\mathrm{mg} .100 \mathrm{~g}^{-1}\right)$ das farinhas de jatobá se encontram na Tabela 3. A FP, como a fração mais comestível do fruto, se destacou por apresentar valor mais expressivo de vitamina C (17,77 mg.100 $\mathrm{g}^{-1}$ ) em relação às outras partes do fruto analisadas, sendo que não diferiram estatisticamente $(p>0,05)$ entre si. A FP apresentou valores próximos ao observado por Cardoso et al. (2013) na polpa do jatobá $\left(8,9 \mathrm{mg} .100 \mathrm{~g}^{-1}\right)$ e em frutas amplamente consumidas pela população brasileira e tidas como fontes de vitamina $\mathrm{C}$, tais como o maracujá $\left(15,60 \mathrm{mg} .100 \mathrm{~g}^{-1}\right)$ 
(OLIVEIRA et al., 2010). O mesmo comportamento da diferença no teor de vitamina $\mathrm{C}$ nas diferentes partes do jatobá, também foi reportado por Dias et al. (2013) que encontraram valores excelentes de vitamina $\mathrm{C}$ para a polpa do jatobá $(121,45$ mg. $\left.100 \mathrm{~g}^{-1}\right)$ e para a semente do fruto $\left(51,87 \mathrm{mg} .100 \mathrm{~g}^{-1}\right)$, teores esses superiores aos destacados aqui.

\section{Composição centesimal das farinhas}

$\mathrm{Na}$ Tabela 4 se encontra os resultados da composição centesimal, valor calórico e fibra bruta da FP, FS e FC. Com relação aos valores obtidos para matéria seca, a FC demonstrou valores mais altos $(94,52 \%)$, evidenciando que a casca possui um teor de umidade menor que as outras partes do fruto (polpa e semente).

Tabela 4. Composição centesimal (média $\pm \mathrm{DP})^{1}$ da farinha da polpa, semente e casca do jatobá-do-cerrado (Hymenaea stigonocarpa Mart.).

\begin{tabular}{|c|c|c|c|}
\hline \multirow{3}{*}{ Constituintes } & \multicolumn{3}{|c|}{ Composição centesimal $\left(\mathrm{g} .100 \mathrm{~g}^{-1}\right)^{*}$} \\
\hline & \multicolumn{3}{|c|}{ Farinhas } \\
\hline & Polpa & Semente & Casca \\
\hline Matéria seca \% & $91,52 \pm 0,23 b$ & $90,51 \pm 0,37 \mathrm{c}$ & $94,52 \pm 0,02 a$ \\
\hline Proteína \% & $7,07 \pm 0,41 b$ & $8,01 \pm 0,16 a$ & $2,08 \pm 0,08 \mathrm{c}$ \\
\hline Cinzas $\%$ & $4,02 \pm 0,02 \mathrm{a}$ & $2,29 \pm 0,08 b$ & $2,25 \pm 0,01 b$ \\
\hline Lipídeos \% & $1,26 \pm 0,27 \mathrm{c}$ & $6,10 \pm 0,85 b$ & $9,77 \pm 0,31 \mathrm{a}$ \\
\hline Carboidratos (excluindo as fibras) $\%$ & $69,83 \pm 3,06 a$ & $73,03 \pm 1,12 \mathrm{a}$ & $47,35 \pm 3,39 b$ \\
\hline Fibra bruta $\%$ & $17,82 \pm 0,21 b$ & $10,57 \pm 0,34 c$ & $38,55 \pm 0,56 a$ \\
\hline Valor Calórico Total (Kcal.100 g $\left.{ }^{-1}\right)$ & $318,94 \pm 0,94 b$ & $379,06 \pm 4,31 \mathrm{a}$ & $285,65 \pm 14,17 \mathrm{c}$ \\
\hline
\end{tabular}

(*) Resultados expressos em base seca $\left(\%=\mathrm{g} .100 \mathrm{~g}^{-1}\right) ;\left({ }^{1}\right)$ Média de três repetições \pm desvio padrão. a, b, c, Letras minúsculas distintas na mesma linha indicam liferença significativa $(\mathrm{p}<0,05)$ pelo teste de Tukey.

Os valores de proteínas (Tabela 4) das farinhas apresentaram diferença estatística $(p<0,05)$, onde a FS obteve os maiores teores $(8,01 \%)$, seguida da FP $(7,07 \%)$ que apresentou resultados superiores, quando comparado com os obtidos por Cardoso et al. (2013) $(5,60 \%$ de proteínas em matéria fresca). Essa divergência pode ser explicada pela origem do material vegetal e pelas condições climáticas e nutricionais de cada região de cultivo (MONCADA et al., 2013). A FC obteve a menor quantidade de proteínas $(2,08 \%)$, abaixo do valor encontrado na casca de buriti (Mauritia flexuosa L.), que foi de 3,20\%, no resíduo analisado por Rodrigues (2010). Porém, a quantidade de proteína das farinhas do presente estudo (FP, FS e FC), foi expressiva em relação a outros frutos do Cerrado, como na polpa do Maracujá BRS Pérolado do Cerrado com $0,95 \%$ de proteínas e, da farinha obtida a partir da casca de Buriti, com 4,64\% de proteínas (VIANA et al., 2016; MORAIS et al., 2019).

Os teores encontrados na FS e FP, possibilitam o seu uso na fortificação protéica de alimentos, contribuindo para a nutrição humana, podendo ainda ser consumida in natura e/ou na aplicação do desenvolvimento de novos produtos alimentícios. Essa estratégia já foi explorada na elaboração de biscoitos tipo cookie, utilizado-se de duas variedades: jatobádo-cerrado (Hymenaea stigonocarpa Mart.) e jatobá-da-mata (Hymenaea stilbocarpa Mart.) e, ainda na elaboração de biscoitos isentos de açúcares, elaborados por Silva et al. (2001).

$\mathrm{O}$ valor de cinzas encontrado na FP foi o mais elevado $(4,02 \%)$ com diferenças estatísticas em relação as demais, o que corrobora com relatos de Cohen (2011), onde o mesmo afirma que a polpa do jatobá apresenta teores expressivos de minerais, destacando-se Ca com 245,3 mg.100 g-1 e o Mg com 194,8 mg.100 g $\mathrm{g}^{-1}$. Valor semelhante de 4,60 e, superior de 5,48\%, foram encontrados por Silva et al. (2001) em polpa do jatobádo-cerrado e jatobá-da-mata, respectivamente. Silva (2013) obteve na farinha deste fruto, um teor de cinzas de 3,50\%, Sousa et al. (2012) reportaram valores de 3,60 e 2,34\% para a polpa farinácea e semente de jatobá, respectivamente. Essa variação no teor de cinzas pode ser atribuída à origem, zona de cultivo (solo, clima) e método de análise pode influenciar nos resultados (COEH, 2010).

Os valores de lipídios (Tabela 4) apresentaram diferença estatística $(\mathrm{p}<0,05)$ entre si, onde a FP $(1,26 \%)$, apresentou menor teor quando comparada as demais (FS $(6,10 \%)$ e FC $(9,77 \%))$ e aos encontrados por Batista et al. (2011) $(3,21 \%)$ e Silva et al. (2001) (entre 2,90 e 3,03\%), para jatoba-do-cerrado e jatoba-da-mata, respectivamente. Por outro lado, quantidade próxima foi avaliada por Silva et al. (2014) (1,72\%). Essa variação pode ser explicada devido as diferentes espécies de jatobás avaliadas em cada estudo citado, conforme reportado por Dias et al. (2013).

O alto valor de lipídeos obtido para a semente em relação à polpa está associado ao princípio de que, nas sementes, encontram-se as reservas nutritivas que servirão ao desenvolvimento do embrião durante a germinação (CHITARRA; CHITARRA, 2005). Além disso, Dias et al. (2013), obtiveram teores médios de $5,78 \%$, estando próximos aos do presente estudo. Consequentemente, a farinha da semente pode ser considerada fonte de lipídeo, possibilitando o seu uso na fortificação de alimentos, além de reduzir custos na produção e o desperdício de resíduos, uma vez que as sementes geralmente não são utilizadas para este fim. Entretanto, a farinha que mais apresentou teor de lipídeos foi a FC $(9,77 \%)$, despertando atenção para esse componente.

Os carboidratos totais, incluindo fibras, apresentaram valor médio de 87,65 (FP); 83,60 (FS) e 85,90\% (FC), com a FP estando próximo ao teor obtido por Batista et al. (2011), de $82,54 \%$ e por Silva et al. (2001), de 83,28\%, além de apresentar-se próximo a outras variedades de jatobá, como o jatobá-da-mata $(83,23 \%)$ também avaliado por Silva et al. (2001).

Dias et al. (2013) relataram teor de $80,95 \%$ de carboidratos nas sementes, teor esse próximo ao valor 
encontrado no presente trabalho $(83,60 \%)$, considerado fonte de energia para a germinação. Já o teor de carboidrato encontrado na FC representa uma boa fonte para desenvolvimento de novos produtos, sendo também uma alternativa de aproveitamento integral do fruto.

Os resultados de fibras totais para as farinhas do jatobá (FP, FS e FC) diferiram significativamente entre si, com a FP $(17,82 \%)$ e, principalmente a FC $(38,55 \%)$ apresentando potencial de aplicação em alimentos que contenham baixo teor de fibras, devido a quantidade expressiva encontrada nessas partes, além de possibilitar o aproveitamente das partes comumente descartadas, como a casca. Dias et al. (2013) relataram que o principal componente da polpa e da semente é a fibra bruta $(50,02$ e 72,14\%, respectivamente), porém em estudo de Dias et al. (2013) não foi avaliado a casca do fruto. Apesar disso, o teor de fibras da FP foi inferior ao obtido por Batista et al. (2011), (23,15\%), sendo que esta disparidade pode estar relacionado a metodologia aplicada, matéria-prima adquirida, estado de maturação, local de cultivo, dentre outros fatores que influenciam os resultados finais obtidos.

Embora a FS apresentando menor menor teor (10,57\%), ainda pode ser utilizado no desenvolvimento de novos produtos, visto que o mesmo apresentou altos teores de proteina e lipídeo, possibilitando o sua utilização.

A FS apresentou conteudo calorico significativamente $(\mathrm{p}<$ 0,05) mais elevado que as FP e FC, podendo-se atribuir principalmente ao baixo teor de fibras totais, assim como ao aumento do teor lipídico da FS (Tabela 4).

Valores diferentes foram observados por Batista et al. (2011), que obtiveram 251,45 Kcal.100 $\mathrm{g}^{-1}$, apresentando resultados inferiores ao do presente trabalho. A heterogeneidade em relação as fibras e ao valor calórico, podem ser explicados pela dos fatores edafoclimáticos de cada região onde as amostras foram adquiridas (SOUSA et al., 2012).

\section{CONCLUSÃO}

Todas as partes do fruto jatobá-do-cerrado têm fontes consideráveis de nutrientes, possibilitando a sua utilização na elaboração de novos produtos alimentícios, pois apresentaram elevados teores de proteínas, carboidratos e fibras.

\section{AGRADECIMENTOS}

Os autores agradecem ao Instituto Federal de Ciência e Tecnologia do Ceará - IFCE - Campus Limoeiro do Norte, à Fundação Cearense de Apoio ao Desenvolvimento Científico e Tecnológico (FUNCAP) e ao Conselho Nacional de Desenvolvimento Científico e Tecnológico (CNPq) pela concessão de bolsa.

\section{REFERÊNCIAS}

ARRUDA, H. S.; FERNANDES, R. V. B.; BOTREL, D. A.; ALMEIDA, M. E. F. Frutos do Cerrado: conhecimento e aceitação de Annona crassiflora Mart. (Araticum) e Eugenia dysenterica Mart. (Cagaita) por crianças utilizando o paladar e a visão. Journal of Health \& Biological Sciences, v. 3, n, 4, p.
224-230,

2015.

10.12662/2317-3076jhbs.v3i4.168.p224-

230.2015

AOAC, ASSOCIATION OF OFFICIAL ANALYTICAL CHEMISTS. Official Methods of Analysis 665 of AOAC International., (2000). CD-ROM. 17th edition, Arlington, VA: AOAC International.

BATISTA, A. G.; ESTEVES, E. A.; DESSIMONI-PINTO, N. A. V.; OLIVEIRA, L. G.; PIRES, S. T.; SANTANA, R. C. Chemical composition of jatobá-do-cerrado (Hymenaea stigonocarpa Mart.) flour and its effect on growth of rats. Alimentos e Nutrição, v. 22, n. 2, p. 173-180, 2011.

CARDOSO, L. DE M. Araticum, cagaita, jatobá, mangaba e pequi do cerrado de Minas Gerais: ocorrência e conteúdo de carotenoides e vitaminas. 2011. 78f. Dissertação (Mestrado em Ciência da Nutrição). UFV, Minas Gerais, 2011.

CARDOSO, L. M.; BEDETTI, S. F.; RIBEIRO, S. M. R.; ESTEVES, E. A.; PINHEIRO-SANT'ANA, H. M. 'Jatoba do cerrado' (Hymenaea stigonocarpa): chemical composition, carotenoidsand vitamins in anexotic fruit from the Brazilian Savannah. Fruits-The International Journal of Tropical and Subtropical Horticulture, v. 68, n. 2, p. 95-107, 2013. $\underline{10.1051 / \text { fruits/2013056 }}$

CHITARRA, M. I. F.; CHITARRA, A. B. Pós-colheita de frutos e hortaliças: fisiologia e manuseio. 2. ed. rev. e ampl. Lavras: UFLA, 2005.

COHEN, K. D. O. Jatobá-do-cerrado: composição nutricional e beneficiamento dos frutos. Planaltina, DF: EMBRAPA: 26 p. 2010 .

CUNHA-SILVA, G. R.; RODRIGUES, C. M.; MIRANDA, S. do C. de. Dados biométricos de frutos e sementes de Hymenaea courbaril var. stilbocarpa (Hayne) Y. T. Lee \& Langenh e H. martiana Hayne. Revista Biotermas, v. 25, n. 3, p. 121-127, 2012. 10.5007/2175-7925.2012v25n3p121

DIAS, L. S.; LUIZA, D. M. M.; JORGE, N. Physicochemical and bioactive properties of Hymenaea courbaril L. pulp and seed lipid fraction. Industrial Crops and Products, v. 49, p. 610 618, 2013. 10.1016/j.indcrop.2013.06.005

FAO. Protein quality evaluation: report of a joint FAO/WHO expert consultation group. Rome, 1990.

FIORDA, F. A.; SIQUEIRA, M. I. D. de. Avaliação do pH e atividade de água em produtos cárneos. Revista Estudos, v. 36, n. 5/6, p. 817-826, 2009.

IAL, Instituto Adolfo Lutz. Métodos físico-químicos para análise de alimentos. 4.ed. Brasília: Ministério da Saúde, 2008. 1020 p.

MACIEL, A. S.; VIEIRA, R. P; GHERARDI, S. R. M. Obtenção e utilização da farinha da casca de jatobá (Hymenae 
courbari) enriquecida com linhaça (Linum usitatissimum) para panificação. Revista Brasileira de Produtos Agroindustriais, v.18, n.1, p. 65-70, 2016.

MENEZES FILHO, A. C. P.; SILVA, M. A.; PEREIRA, A. V.; OLIVEIRA FILHO, J. G.; CASTRO, C. F. S. Parâmetros físico-químicos, tecnológicos, atividade antioxidante, conteúdo de fenólicos totais e carotenóides das farinhas dos frutos do jatobá-do-cerrado (Hymenaea stigonocarpa Mart. ex Hayne). Multi-Science Journal, v. 2, n. 1, p. 93-100, 2019. 10.33837/msj.v2i1.900.

MONCADA, A.; MICELI, A.; VETRANO, F.; MINEO, V.; PLANETA, D.; D'ANNA, F. Effect of grafting on yield and quality of eggplant (Solanum melongena L.) Scientia Horticulturae. v. 149, p. 108-114, 2013. $\underline{10.1016 / j . s c i e n t a .2012 .06 .015}$

MORAIS, R. A.; MELO, K. K. S.; OLIVEIRA, T. T. B. Chemical, physical and technological characterization of fish meal from Buriti shell (Mauritia flexuosa L. f.). Brazilian Journal of Development, Curitiba, v. 5, n. 11, p. 23307-23322, nov. 2019. 10.34117/bjdv5n11-050

OLIVEIRA, D. S.; LOBATO, A. L.; RIBEIRO, S. M.; SANTANA, A. M.; CHAVES, J. B.; PINHEIRO, S. H. M. Carotenoids and Vitamin $\mathrm{C}$ during Handling and Distribution of Guava (Psidium guajava L.), Mango (Mangifera indica L.), and Papaya (Carica papaya L.) at Commercial Restaurants. Journal of Agricultural and Food Chemistry, v. 58, n. 10, p. 6166-6172, 2010. 10.1021/jf903734x.

PEREIRA, M. M.; OLIVEIRA, E. N. A. de.; ALMEIDA, F. L. C.; FEITOSA, R. M. Processamento e caracterização físicoquímica de biscoitos amanteigados elaborados com farinha de jatobá. Revista Brasileira de Tecnologia Agroindustrial, v. 10, n. 2, p. 2137-2149, 2016.

PUGA, I. T. Obtenção de produtos naturais a partir da casca do fruto do jatobá (Hymenaea courbaril). 55 f. 2015. Trabalho de conclusão de curso (Especialização em Química Tecnológica) Universidade de Brasília, Brasília - DF, 2015.

R CORE TEAM. R: A language and environment for statistical computing. R Foundation for Statistical Computing, Vienna, Austria, 2018.

RAMOS, F. S. A. R.; SANTOS, T. C.; FERREIRA, T. H. B.; GOMES, M. C. S.; MUNHOZ, C. L. Aceitabilidade de Biscoito Tipo Cookie Enriquecidos com Farinha de Jatobá. Cadernos de Agroecologia, v. 13, n. 2, p. 1-7, 2018.

RODRIGUES, B. S. Resíduos da agroindústria como fonte de fibras para elaboração de pães integrais. 2010. 96f. Dissertação (Mestrado em Ciência e Tecnologia de Alimentos) Universidade de São Paulo: Escola Superior de Agricultura "Luiz de Queiroz”, São Paulo, 2010.
SILVA, C. P da.; SOUSA, M. S. B.; SIGUEMOTO, E. S.; SOARES, R. A. M.; ARÊAS, J. A. G. Chemical composition and antioxidant activity of jatobá-do-cerrado (Hymenaea stigonocarpa Mart.) flour. Food science and technology, v. 34, n. 3 , p. 597-603, 2014. 10.1590/1678-457x.6405

SILVA, C. P. da. Efeito da adição de farinha de jatobá-docerrado (Hymenaea stigonocarpa Mart.) na resposta glicêmica de pães. 2013. 77f. Dissertação (Mestrado em Nutrição em Saúde Pública). USP, SP, 2013.

SILVA, C. P. MANOLIO, S. F. R. A.; SAMPAIO, G. R.; BARROS, M. C. S.; do NASCIMENTO, T. P.; CAMERON, L. C.; LARRAZ, M. S. F.; GOMES, J. A. A. Identification and action of phenolic compounds of Jatobá-do-cerrado (Hymenaea stignocarpa Mart.) on $\alpha$-amylase and $\alpha$-glucosidase activities and flour effect on glycemic response and nutritional quality of breads. Food Research International, v. 116, p. 1076-1083, 2019. 10.1016/j.foodres.2018.09.050.

SILVA, M. R.; SILVA, M. S.; MARTINS, K. A.; BORGES, S. Utilização tecnológica dos frutos de jatobá-do-cerrado e de jatobá-da-mata na elaboração de biscoitos fontes de fibra alimentar e isentos de açúcares. Revista Ciência e Tecnologia de Alimentos, v. 21, n. 2, p. 176-182, 2001. 10.1590/S010120612001000200010.

SILVA, R. S.; PEREIRA, E. M.; COSTA, J. C.; OLIVEIRA, F. L. N.; AZERÊDO, G. A. Qualidade físico-química de farinhas de genótipos de berinjela (Solanum melongena L.) submetidas ao cultivo orgânico. Revista Verde de Agroecologia e Desenvolvimento Sustentável, v.11, n 3, p. 59-64, 2016.

SOUSA, E. P. de.; SILVA, L. M. de M.; SOUZA, F. C. de.; FERRAZ, R. R; FAÇANHA, L. M. Caracterização físicoquímica da polpa farinácea e semente do jatobá. Revista Verde de Agroecologia e Desenvolvimento Sustentável, v.7, n. 2, p. 117-121, 2012.

STROHECKER, R; HENNING, H. M. Analisis de vitaminas: métodos comprobados. Madrid: Paz Montalvo. 1967, 428 p.

VIANA, M. L.; COSTA, A. M.; CELESTINO, S. M. Informações para a composição de tabela nutricional da polpa do maracujá BRS Pérola do Cerrado. (Boletim de Pesquisa e Desenvolvimento/Embrapa Cerrados) Planaltina, DF: Embrapa Cerrados, 2016. 17 p. 